\title{
The ANNIE Experiment
}

\author{
Francesca Di Lodovico*i \\ Queen Mary University of London \\ E-mail: f.di.lodovico@gmul.ac.uk
}

The Accelerator Neutrino Nucleon Interaction Experiment (ANNIE) at Fermilab is designed to measure the neutron yield of neutrino interactions in gadolinium-doped water using accelerator beam events. An innovative aspect of the ANNIE design is the use of precision timing to localize interaction vertices in the small fiducial volume of the detector using early prototypes of LAPPDs (Large Area Picosecond Photodetectors). This experiment will be a first application of these devices demonstrating their feasibility for water Cherenkov neutrino detectors and could have a transformative impact on water Cherenkov and other photodetection-based neutrino detector technologies.

16th International Workshop on Neutrino Factories and Future Neutrino Beam Facilities 25 -30 August, 2014

University of Glasgow, United Kingdom

\footnotetext{
* Speaker.

${ }^{\dagger}$ Partially supported by the grant ERC-207282.
} 

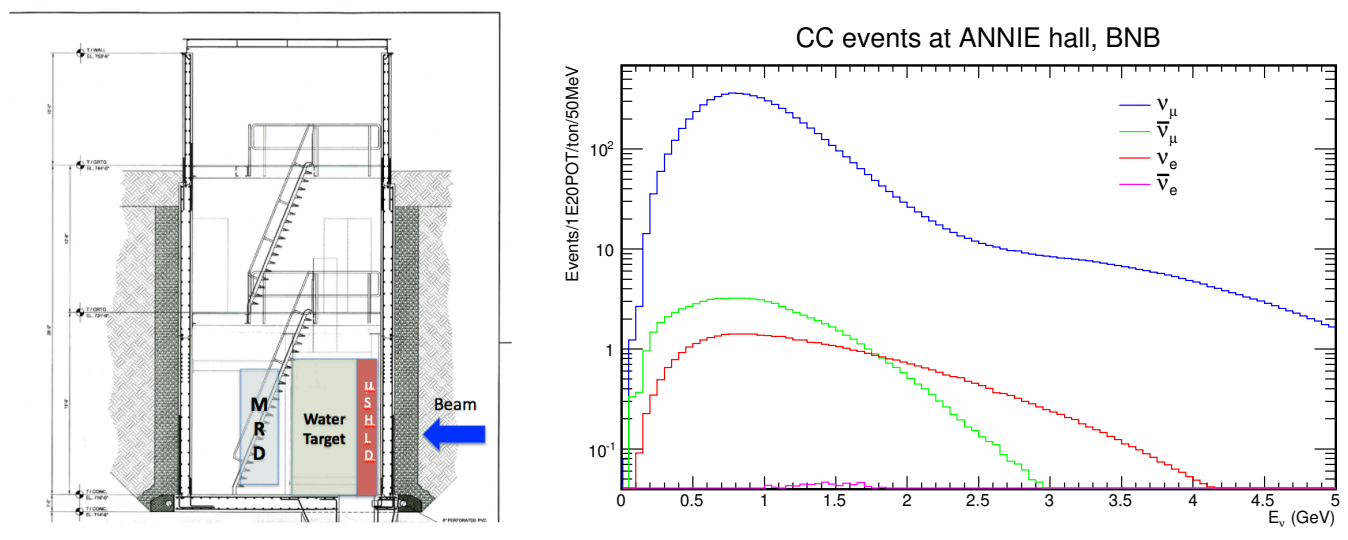

Figure 1: Left figure: ANNIE in the SciBooNE hall. Right figure: neutrino flux spectra expected in the SciBooNE hall from the Booster Neutrino Beam.

\section{Introduction}

The ability to detect final state neutrons from neutrino nucleon interactions is crucial to understand the nuclear process that occurs when the neutrino interacts with the nucleon. The observation of neutron multiplicity can distinguish the different nuclear processes.

Tagging events by the presence and number of final-state neutrons can provide physics analyses with a better handle for signal-background separation and even allow for discrimination between different varieties of neutrino interactions. A promising technique for detecting final state neutrons is the search for a delayed signal from their capture on Gadolinium dissolved in water.

ANNIE [1], the Accelerator Neutrino Nucleon Interaction Experiment, is a Gd-doped water Cherenkov (WC) target in front of a muon range detector (MRD) sufficiently instrumented to be able to stop and detect the gammas from the neutron capture in gadolinium.

Using ANNIE, we propose to make a systematic measurement of the neutron yield from neutrino interactions of energies around $700 \mathrm{MeV}$ using the Booster Neutrino Beam at Fermilab. The experiment will be located in the existing "SciBooNE" hall [2], which sits in a prime experimental location on the FNAL booster beamline (Fig. 1, left plot).

In the following, we will first describe the experiment, then the neutron capture in gadolinium and finally the physics goals and expected plan for the different phases of ANNIE. The first phase of ANNIE was approved by the Fermilab Physics Advisory Committee in January 2015.

\section{The ANNIE Experiment}

The Gd-doped WC tank of the ANNIE detector, complemented by a muon range detector downstream, will be instrumented with Large Area Picosecond Photodetectors (LAPPDs) [3] for detailed event reconstruction and a large number of conventional photomultiplier tubes (PMTs) to improve the light collection for energy reconstruction and efficient neutron tagging.

The footprint for the water target is essentially that of the SciBooNE detector, a cylindrical volume roughly $3.8 \mathrm{~m}$ long and $2.3 \mathrm{~m}$ in diameter. 


\begin{tabular}{|l|c|c|c|}
\hline $\boldsymbol{v}$-type & Total Interactions & Charged Current & Neutral Current \\
\hline \hline$v_{\mu}$ & 9892 & 6991 & 2900 \\
\hline $\bar{v}_{\mu}$ & 130 & 83 & 47 \\
\hline$v_{e}$ & 71 & 51 & 20 \\
\hline $\bar{v}_{e}$ & 3.0 & 2.0 & 1.0 \\
\hline
\end{tabular}

Table 1: Rates expected in 1 ton of water with $1 \times 10^{20}$ POT exposure at the SciBooNE hall.

The Booster Neutrino Beam, that ANNIE will use, will run at $7.5 \mathrm{~Hz}$, with roughly $4 \times 10^{12}$ protons-on-target (POT) per spill. These are delivered in 81 bunches over a $1.6 \mu$ s spill time to a target and horn combination $100 \mathrm{~m}$ upstream of the SciBooNE hall. This beam is about $93 \%$ pure $v_{\mu}$ (when running in neutrino mode) and has a spectrum that peaks at about $700 \mathrm{MeV}$ (Fig. 1, right plot). We expect on the order of 7,000 charged current muon neutrino interactions per ton per $10^{20}$ POT over a period of 6 months. The neutrino rates are shown in Table 1.

The events will be collected by ANNIE using a hybrid of LAPPDs and conventional PMTs. In order to maximize its light collection, ANNIE needs to augment its LAPPD coverage with an existing stock of conventional 8-inch PMTs (manufactured by Hamamatsu) taken from the IMB and Super-Kamiokande (Super-K) experiments.

The Large Area Picosecond Photodetector project was formed to develop new fabrication techniques for making large-area (8" x 8") Multichannel Plate photodetectors using low-cost materials, well established industrial batch techniques, and advances in material science. LAPPDs may be a transformative technology for WC detectors. While conventional photomultipliers are singlepixel detectors, LAPPDs are imaging tubes, able to resolve the position and time of single incident photons within an individual sensor. This maximizes the usage of the fiducial volume as it allows for reconstructing events very close to the wall of the detector, where the light can only spread over a small area. The simultaneous time and spatial resolutions of the LAPPDs, at better than 100 picoseconds and $3 \mathrm{~mm}$ for single photons, represent a major improvement over conventional PMTs. Preliminary Monte Carlo studies indicate that the measurement of Cherenkov photon arrival space-time points with resolutions of $1 \mathrm{~cm}$ and $100 \mathrm{psec}$ will allow the detector to function as a tracking detector, with track and vertex reconstruction approaching size scales of just a few centimeters [4]. Imaging detectors would enable photon counting by separating between the space and time coordinates of the individual hits, rather than simply using the total charge. This means truly digital photon counting and would translate directly into better energy resolution and better discrimination between dark noise and photons from neutron captures. Finally, at a thickness of less than $1.5 \mathrm{~cm}$, LAPPDs maximize the use of the limited fiducial volume available to small detectors.

The DOE awarded \$3M through the STTR program to Incom, Inc to develop a commercial line for the LAPPDs over the next several years. The number of LAPPDs available to ANNIE will then increase with time, as more LAPPDs become available through the commercialization process. 


\section{Using Gadolinium}

Even moderately energetic neutrons ranging from tens to hundreds of $\mathrm{MeV}$ will quickly lose energy by collisions with free protons and oxygen nuclei in water. The cross sections for these capture reactions are 0.33 barns and 0.19 millibarns, respectively, so to first approximation every thermal neutron is captured on a free proton via the reaction $n+p \rightarrow d+\gamma$. The resulting gamma has an energy of $2.2 \mathrm{MeV}$ [5] and makes very little detectable light since the Compton scattered electron is close to the Cherenkov threshold. The entire sequence from liberation to capture takes around $200 \mu$, with only a very small dependence (plus or minus a few $\mu$ s) on initial neutron energy.

The situation is much improved by adding a water-soluble gadolinium compound, gadolinium chloride, $\mathrm{GdCl}_{3}$, or the less reactive though also less soluble gadolinium sulfate, $\mathrm{Gd}_{2}\left(\mathrm{SO}_{4}\right)_{3}$, to the water. Naturally occurring gadolinium has a neutron capture cross section of 49,700 barns, and these captures produce an $8.0 \mathrm{MeV}$ gamma cascade. The visible energy will be around $4-5 \mathrm{MeV}$ and thus visible in a WC detector. Due to the much larger cross section of gadolinium, adding just $0.2 \%$ of one of these compounds (about $0.1 \% \mathrm{Gd}$ ) by weight is sufficient to cause $90 \%$ of the neutrons to capture visibly on gadolinium instead of invisibly on hydrogen. Following the addition of gadolinium the time between neutron liberation and capture is reduced by an order of magnitude to around $20 \mu$ s, greatly suppressing accidental backgrounds.

A major limitation on the effective execution of neutron tagging techniques comes from large uncertainties on the nuclear mechanisms that produce neutrons and consequently on how many neutrons are produced by high energy ( $\mathrm{GeV}$-scale) neutrino interactions. The number of neutrons is expected to depend on the type of neutrino interaction and on the momentum transfer with higher energy interactions producing more than one neutron. The exact number of neutrons is determined by a variety of nuclear processes but, if a typical category of events consistently produce more than one neutron, the absence of any neutron would significantly increase the confidence in that category of events.

The measurement of the neutron multiplicity in neutrino nucleon interactions by ANNIE will result in:

- a better understanding of the nuclear reactions in the neutrino nucleon interactions that will help to improve the current models used in the generators;

- an improved knowledge of the proton decay background. Detailed nuclear calculations by Ejiri [6] predict that only $8 \%$ of proton decays in oxygen will result in neutron emission. This means that only approximately $6 \%$ ( $8 \%$ of $80 \%)$ of all proton decays in water should result in neutrons (ignoring FSI production by proton decay daughters). Therefore neutron tagging to reject atmospheric neutrino backgrounds incurs a modest loss of signal efficiency (see e.g. in Fig. 2, left plot, the possible benefit of this method).

- implications for accelerator physics: helps in selecting the signal CCQE events and reduce the background, this will drastically improve the signal measurement both at the far and near detectors.

- a better understanding the processes related to supernova neutrinos. The main detection channel for supernova relic neutrinos in water Cherenkov detectors that comes from positrons 
emitted by inverse $\beta$ decay reactions and the neutron capture in gadolinium helps to tag these events.

\section{Event Reconstruction}

Neutrino interactions in the water target will produce a flash of light with no signal in a front anti-coincidence counter. Events can be selected so that they are "CCQE-like", i.e., there is a single muon track in the MRD that points back to the rough position of the vertex in the target. Following a valid CCQE candidate, neutron capture events must be detected in the target for about $100 \mu \mathrm{s}$, or about three capture times. If the vertex is restricted to the central volume of the water target, then there are several hadron scattering lengths in all directions, which should be enough to slow down and stop neutrons in the range of $110 \mathrm{MeV}$. Higher energy neutrons could require external counters.

The vertex of each neutrino can be reconstructed using several different approaches. The ideal method is to fit for the earliest light emitted along the muon track, using the track parameters extracted from the muon range detector. It may be possible to use the isotropic light emitted from the neutron captures themselves to determine where in the volume the captures are happening. By measuring the muon direction to a precision of roughly $10^{\circ}$ and the muon momentum (from a range measurement) to roughly $20 \%$, we will be able to accurately reconstruct the multiplicity as a function of the momentum transfer to the nucleus from the neutrino.

While basic simulations of the ANNIE detector already exist, we are in the process of building a fully integrated Geant 4 simulation of the experiment. Through these simulations, we can address the technical design issues required for the success of this effort.

\section{Sensitivities Using ANNIE}

The main deliverable from this experiment is a measurement of the final-state neutron abundance as a function of momentum transfer from charged current neutrino interactions. This measurement is similar to that shown in Fig. 2 (right plot) by the Super-K collaboration, except that we would reconstruct the total momentum transfer rather than visible energy. The ANNIE detector will be optimized for efficient detection of captured neutrons produced in its fiducial volume using the gadoliunium and the LAPPDs. Furthermore, it may be possible to separate the data between a variety of charged current event types and possibly neutral current interactions.

To first order, neutrino interactions with nucleii will predictably yield either 1 or 0 neutrons in the final state: neutrinos interacting by charged current exchange will produce a final-state proton and no additional neutrons, whereas anti-neutrinos produce exactly one final-state neutron. High energy neutral current interactions tend to produce either protons or neutrons, proportional to the abundance of each nucleon in water.

Moreover, GeV-scale (anti-)neutrinos can produce additional neutrons through the complex interplay of higher-order and multi-scale nuclear physics:

- secondary $(\mathrm{p}, \mathrm{n})$ scattering of struck nucleons within the nucleus;

- charge exchange reactions of energetic hadrons in the nucleus (e.g., $\pi^{-}+p \rightarrow n+\pi^{0}$ ); 

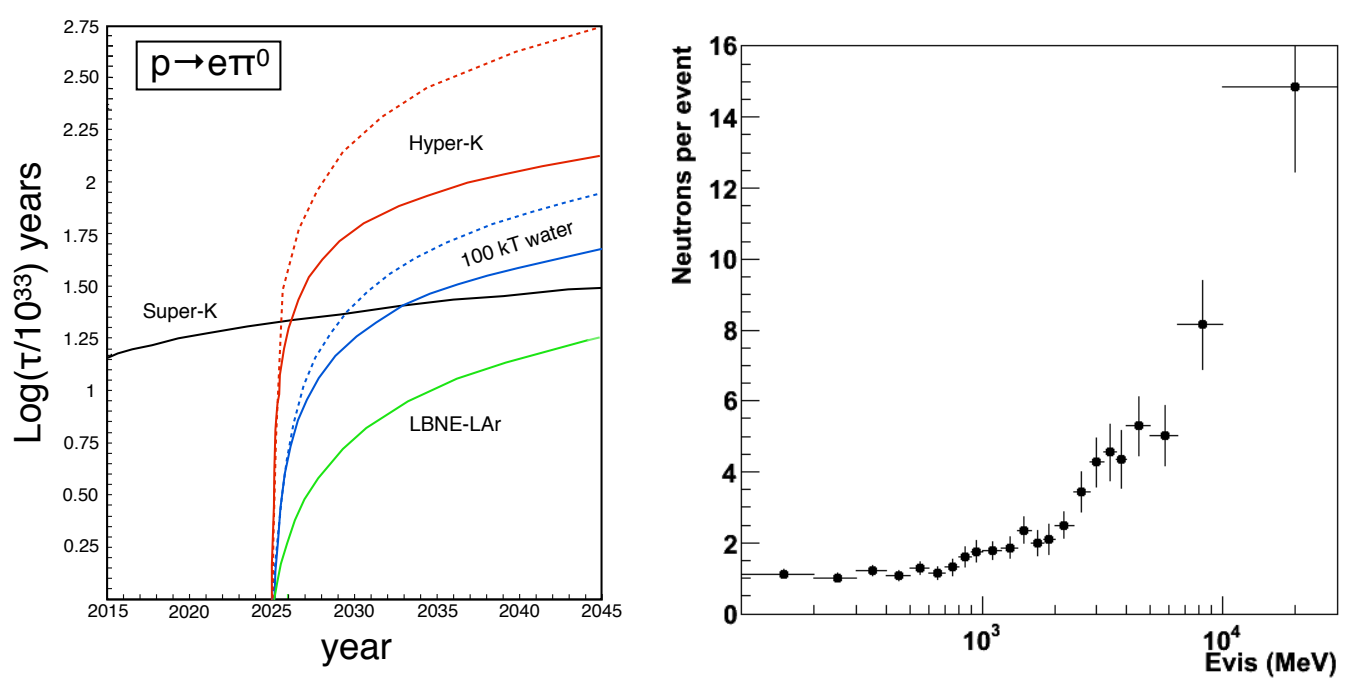

Figure 2: Left figure: proton decay sensitivity in the $p \rightarrow e+\pi_{0}$ channel for Super-K (black), Hyper-K (red), the LBNF 34-kton LAr detector (green), and a hypothetical 100-kton water volume (blue). For Hyper$\mathrm{K}$ and the 100 kton water volume two scenarios are presented: sensitivity including atmospheric neutrino backgrounds (solid) and limits and sensitivity with $90 \%$ of backgrounds removed (dashed). Right figure: measurement of neutron multiplicity in pure water versus visible energy by the Super-K collaboration [7].

- de-excitation by neutron emission of the excited daughter nucleus;

- capture of $\pi^{-}$events by protons in the water, or by oxygen nuclei, followed by nuclear breakup;

- meson exchange currents, where the neutrino interacts with a correlated pair of nucleons, rather than a single proton or neutron;

- secondary neutron production by proton or neutron scattering in water.

Consequently, neutron multiplicity distributions tend to peak at 0 or 1 with long tails. Given the highly non-gaussian shape of these distributions, parameters such as the mean neutron yield are not necessarily illuminating. At the simplest level, we want to measure $\mathrm{P}(\mathrm{N}=0), \mathrm{P}(\mathrm{N}=1)$, and $\mathrm{P}(\mathrm{N}>1)$ with particular attention to any excesses beyond tree-level expectations. These measurements, binned by interaction type and kinematics, will provide a strong handle to constrain nuclear models, even in the absence of detailed shape information beyond $\mathrm{P}(\mathrm{N}=2)$.

When using the presence of final state neutrons to separate experimental backgrounds in various physics analyses, the shape of the far tail becomes increasingly less important with higher N. For example, in the case of proton decay we are interested in the efficiency for detecting any neutrons at all. The rate for atmospheric neutrinos faking a proton decay $(f)$ is given by:

$$
f=P(0)+P(1)(1-\varepsilon)+P(2)(1-\varepsilon)^{2}+P(3)(1-\varepsilon)^{3}+\ldots
$$


where $\mathrm{P}(\mathrm{N})$ is the probability of $\mathrm{N}$ neutrons given a background event, and $\varepsilon$ is the neutron detection efficiency. For high neutron detection efficiencies such as the expected $68 \%$ in a Gdloaded Super-K fill, higher order terms quickly drop off, and $f$ can be accurately estimated by the integral of $\mathrm{P}(\mathrm{N}>2)$ without any further shape information.

\section{Timeline}

The operation of the experiment is planned in three phases.

- Phase I: Technical Development and Background Characterization - year 1.

A variety of background neutrons, both those originating from rock, and those from sky shine, present a challenge for ANNIE. Previous studies on these backgrounds are available in Refs. $[8,9,10,11]$. Characterization of these background neutrons can occur before full LAPPD coverage is available. This represents an opportunity to make relevant physics and technical measurements while testing and developing the technology for the full physics experiment, in the second and third phases. This first phase of the experiment was approved by the Fermilab Physics Advisory Committee in January 2015 with expected installation by the end of 2015.

- Phase II: ANNIE physics run I - year 2.

Phase II of ANNIE would represent the first full physics run. This phase will begin when we have acquired sufficient LAPPDs to operate our "low-coverage" LAPPD scenario, as determined by simulation and reconstruction studies.

This first measurement will be an inclusive measurement of neutron yields as a function of reconstructed transferred moment, $q^{2}$, and visible energy, $E_{v i s}$, with the possibility of separating between charged current and neutral current events. We will also test, with limited coverage, the ability to identify neutrino interactions that imitate proton decays.

- Phase III: ANNIE physics run II - year 3.

Phase III represents the full realization of the ANNIE detector. We estimate that this will correspond to roughly 50-100 LAPPDs. With sufficient LAPPD coverage, detailed kinematic reconstruction and particle ID may become possible. In such a case, we will measure neutron yields for event classes separated by detailed inventory of final state particles.

After successful operation of the two ANNIE physics runs to the satisfaction of the collaboration, additional runs may be possible with the goal of improving on the physics and testing new R\&D directions. These directions might include tests with water-based Liquid Scintillator.

\section{Conclusions}

The ANNIE experiment provides an opportunity to make an important measurement of the final-state neutron abundance from neutrino interactions with water nuclei as a function of momentum transfer. This measurement will have a significant impact on a variety of future physics 
analyses, including a nearly factor of five (or more) improvement in the sensitivity of WC proton decay searches provided by efficient neutron tagging. ANNIE will represent a first demonstration detector for WC reconstruction using the newly developed, high resolution LAPPD imaging sensors.

\section{References}

[1] I. Anghel et al. [ANNIE Collaboration], Expression of Interest: The Atmospheric Neutrino Neutron Interaction Experiment (ANNIE), arXiv:1402.6411 [physics.ins-det].

[2] K. Hiraide et al [SciBooNE Collaboration], Search for charged current coherent pion production on carbon in a few-GeV neutrino beam, Phys. Rev. D 78 (2008) 112004.

[3] The Large Area Picosecond Photodetector Collaboration: http://psec.uchicago.edu

[4] I. Anghel presenting work by I. Anghel, E. Catano-Mur, M.C. Sanchez, M. Wetstein, and T. Xin at DPF 2013; arXiv:1310.2654 [physics.ins-det].

[5] R.E. Meads et al, The capture cross section of thermal neutrons in water, Proc. Phys. Soc. A 69 (1956) 469.

[6] H. Ejiri, Nuclear deexitations of nucleon holes associated with nucleon decays in nuclei, Phys. Rev. C 48 (1993) 1442-1444.

[7] H. Zhang for the [Super-Kamiokande Collaboration], Neutron tagging and its physics application in Super-Kamiokande-IV, Proceedings of the 32 International Cosmic Ray Conference, Beijing (2011).

[8] W. Lee et al., Phys. Rev. Lett. 37, 186 (1976).

[9] Bartol neutron monitor data, http://neutronm.bartol.udel.edu/. Neutron monitors of the Bartol Research Institute are supported by NSF grant ATM- 0000315.

[10] A. A. Aguilar-Arevalo et al. [SciBooNE Collaboration], Bringing the SciBar detector to the booster neutrino beam, arXiv:0601022 [hep-ex].

[11] H. Takei for the SciBooNE Collaboration, Identification of Recoil Proton Tracks for a Neutrino Neutral Current Elastic Scattering Cross-Section Measurement at SciBooNE, talk at the 2008 Meeting of the American Physical Society. 